\title{
AM-SIGNAL DETECTION IN COGNITIVE RADIOS USING FIRST-ORDER CYCLOSTATIONARITY
}

\author{
Yi Zhou, Khalid Qaraqe, and Erchin Serpedin* \\ Dept. of Electrical \& Computer Engineering \\ Texas A\&M University \\ College Station, TX 77843-3128, USA
}

\author{
Octavia A. Dobre
}

\author{
Faculty of Eng. \& Applied Science \\ Memorial University of Newfoundland \\ St. John's, NL A1B 3X5, Canada
}

\begin{abstract}
Cognitive radio is regarded as a novel approach for improving the utilization of precious radio spectrum resource. The detection of very low signal-to-noise ratio (SNR) signals with relaxed a priori information on the signal parameters is of high importance to such radios. This paper proposes a detection algorithm based on the first-order cyclostationarity for amplitude modulated (AM) signals that only requires rough information on the signal bandwidth and carrier frequency. A theoretical asymptotic analysis is performed. Simulation results show that the proposed algorithm performs well at low SNRs.
\end{abstract}

Index Terms - Cognitive Radio, Cyclostationarity, Signal Detection, Amplitude Modulation

\section{INTRODUCTION}

The need for higher data rates is increasing as a result of the transition from conventional wireless voice-oriented communications to wireless multimedia applications. Given the scarcity of the usable frequency spectrum, it turns out that static frequency allocation schemes cannot accommodate the need for increased data rates. Therefore, new ways for exploiting the available spectrum are needed. Cognitive radios represent intelligent wireless communication systems capable of sensing and adapting their features to the existing radio frequency environment [1]. The most important component of cognitive radios is their ability to measure, sense and be aware of the radio channel characteristics, detect availability of spectrum and received signal features (power, modulation) to opportunistically search for and exploit the unoccupied spectrum [2] [3]. Since a significant portion of the spectrum allocated to licensed services is sparsely occupied at any given time, a dynamic spectrum allocation strategy can yield major benefits. Notice also that intelligent radios are also required for real-time signal interception and processing in many other applications. A major issue in cognitive

*Dr. E. Serpedin is the corresponding author. E-mail: serpedin@ece.tamu.edu radios is the detection of signals of very low signal-to-noise ratio (SNR) assuming as little as possible a priori information about the signal parameters.

In this paper, first-order cyclostationarity of band-limited amplitude modulated (AM) signals that are affected by additive Gaussian noise, carrier phase and frequency offset, and time delay is investigated for detection. A first-order cyclic moment based feature is proposed, which requires only rough information on the signal bandwidth and carrier frequency. An algorithm is further proposed for detection of the AM signal. Theoretical analysis for the asymptotic statistics of the first-order cyclic moment is conducted. The rest of the paper is organized as follows. The signal model and the first-order cyclostationarity are introduced in Section 2 . The asymptotic analysis for the cyclostationary statistics of the signals of interest is performed in Section 3. Section 4 proposes a detection algorithm based on the first-order cyclic moment feature for the AM signal and the performance analysis is carried out therein. Section 5 concludes the paper.

\section{FIRST-ORDER CYCLOSTATIONARITY OF AMPLITUDE MODULATED SIGNALS}

\subsection{Signal Model}

Assuming rough information on the signal bandwidth and carrier frequency, the out-of-band noise and interferences are removed by appropriate filtering and the received signal is then down-converted, yielding the signal model:

$$
r(t)=x(t)+\nu(t),
$$

where $x(t)$ represents an amplitude modulated (AM) signal, which is affected by phase, frequency offset and time delay, and $\nu(t)$ denotes the additive zero-mean Gaussian noise. The signal of interest $x(t)$ is expressed as

$$
x(t)=A e^{j \theta} e^{j 2 \pi \Delta f t}\left(1+\eta u\left(t-t_{0}\right)\right),
$$

where $A$ is the signal amplitude, $\theta$ stands for the carrier phase offset, $\Delta f$ denotes the carrier frequency offset, $t_{0}$ is the time delay, $\eta$ denotes the modulation index and $u(t)=m(t) * g(t)$ 
with $m(t)$ denoting normalized zero-mean real-valued modulating signal and $g(t)$ representing the impulse response of the low-pass receive filter. In addition, the modulation constraint $|\eta u(t)|<1$ is assumed satisfied.

\subsection{First-Order Cyclostationary Statistics}

The received signal is sampled at the output of mixer with the sampling rate $f_{s}=1 / T_{s}$ yielding the discrete-time first-order cyclostationary signal

$$
r[n]=\left.r(t)\right|_{t=n T_{s}} .
$$

The signal $r[n]$ is a discrete-time first-order cyclostationary process, and its first-order time-varying moment $\mu_{r}[n]=$ $\mathbf{E}\{r[n]\}$ is an almost periodic function of time which admits the Fourier series expansion [4]

$$
\mu_{r}[n]=\sum_{\alpha \in \kappa} m_{r}(\alpha) e^{j 2 \pi \alpha n}
$$

where $\kappa=\left\{\alpha: m_{r}(\alpha) \neq 0\right\}$ represents the set of first-order cyclic frequencies (CFs) and $m_{r}(\alpha)$ denotes the first-order cyclic moment (CM) at $\mathrm{CF} \alpha$ defined as

$$
m_{r}(\alpha)=\lim _{N \rightarrow \infty} \frac{1}{N} \sum_{n=0}^{N-1} \mu_{r}[n] e^{-j 2 \pi \alpha n} .
$$

The estimator of the first-order CM at CF $\alpha$ based on $N$ samples is given by [5]

$$
\hat{m}_{r}^{(N)}(\alpha)=\frac{1}{N} \sum_{n=0}^{N-1} r[n] e^{-j 2 \pi \alpha n}
$$

\section{ASYMPTOTIC ANALYSIS}

In this section, the asymptotic statistic of the first-order CM in the presence of a large observation size is presented. Based on the asymptotic analysis, we propose a detection algorithm for AM signals in the next section.

\subsection{Asymptotic Statistics for AM Signals}

When an AM signal is present at the receiver, ignoring the noise, the sampled received signal can be expressed as

$$
r[n]=A e^{j \theta} e^{j 2 \pi \Delta f T_{s} n}(1+\eta u[n]),
$$

where $u[n]=u\left(n T_{s}-t_{0}\right)$.

Given $N$ samples, the estimator of the first-order CM of the AM signal at $\mathrm{CF} \alpha$ takes the form

$$
\begin{aligned}
\hat{m}_{r}^{(N)}(\alpha) & =\frac{1}{N} \sum_{n=0}^{N-1} r[n] e^{-j 2 \pi \alpha n} \\
& =\frac{A e^{j \theta}}{N} \sum_{n=0}^{N-1} e^{j 2 \pi\left(\Delta f T_{s}-\alpha\right) n}(1+\eta u[n])
\end{aligned}
$$

Since $\mathbf{E}\{u[n]\}=0$,

$$
\mathbf{E}\left\{\hat{m}_{r}^{(N)}(\alpha)\right\}=\frac{A e^{j \theta}}{N} \sum_{n=0}^{N-1} e^{j 2 \pi f_{0} n}
$$

where $f_{0}=\Delta f T_{s}-\alpha$. By applying the definition of Kronecker's delta

$$
\lim _{N \rightarrow \infty} \frac{1}{N} \sum_{n=0}^{N-1} e^{j 2 \pi f_{0} n}=\delta\left(f_{0}\right)=\left\{\begin{array}{ll}
1 & f_{0}=0 \\
0 & \text { else }
\end{array},\right.
$$

it follows that

$$
\lim _{N \rightarrow \infty} \mathbf{E}\left\{\hat{m}_{r}^{(N)}(\alpha)\right\}=A e^{j \theta} \delta\left(f_{0}\right) .
$$

Therefore, one can find that the asymptotic mean of the firstorder CM is non-zero only at the CF $\alpha=\Delta f T_{s}$.

Based on Eqs. (2) and (3), one can infer the asymptotic variance of the first-order CM as follows

$$
\lim _{N \rightarrow \infty} N \operatorname{var}\left\{\hat{m}_{r}^{(N)}(\alpha)\right\}=A^{2} \eta^{2} S_{u u}\left(f_{0}\right)
$$

where $S_{u u}\left(f_{0}\right)$ represents the power spectral density of the modulating signal $u[n]$ at frequency $f_{0}$.

Next we investigate the asymptotic normality of $\hat{m}_{r}^{(N)}(\alpha)$. Along the lines of [5], without any loss of generality we can make the assumption that the signal $\{r[n]\}$ satisfies the following mixing condition.

Assumption (A1): $\{r[n]\}$ is a random sequence such that all its cumulants of all orders satisfy the condition: for $m \in \mathbf{Z}$ and $l \in\{1, \cdots, m\}$

$$
\sum_{\xi_{1} \cdots \xi_{m}=-\infty}^{\infty} \sup _{\substack{n \\ \text { ( }}}\left|\xi_{l} \operatorname{cum}\left\{r[n], r\left[n+\xi_{1}\right], \cdots, r\left[n+\xi_{m}\right]\right\}\right|<\infty .
$$

Intuitively, Assumption (A1) implies that the samples of the process $\{r[n]\}$ that are well separated in time are approximately independent. Mixing conditions of this form were proposed also by [6] for developing consistent polyspectrum estimators for stationary processes and are met by AM signals as is the case with the present scenario.

Following the lines of proof in [5] [7], it can be shown that the asymptotic normality of $\hat{m}_{r}^{(N)}(\alpha)$ follows since the cumulants of order $\geq 3$ of $\hat{m}_{r}^{(N)}(\alpha)$ vanish asymptotically. Therefore, for large enough sample size $N, \hat{m}_{r}^{(N)}(\alpha)$ asymptotically follows a complex normal distribution $\mathcal{C N}\left(A e^{j \theta}, A^{2} \eta^{2}\right.$ $\left.S_{u u}(0) / N\right)$ at CF $\alpha=\Delta f T_{s}$. As a corollary, it follows that the estimator given in Eq. (1) is consistent.

\subsection{Cyclostationary Statistics of Noise}

The noise $\nu[n]$ is the output of the receive low-pass filter, and assume the following expression

$$
\nu[n]=\sum_{i=-\infty}^{\infty} w[n-i] g[i]
$$


where $\{w[n]\}$ are i.i.d. complex Gaussian random variables with zero mean and variance $\sigma^{2}$. It follows that

$$
\mathbf{E}\left\{\nu[n] \nu^{*}[m]\right\}=\sigma^{2} \sum_{k=-\infty}^{\infty} g[k] g[n-m+k]
$$

The variance of the cyclostationary moment estimator is given by

$$
\operatorname{var}\left\{\hat{m}_{r}^{(N)}(\alpha)\right\}=\frac{1}{N^{2}} \sum_{n=0}^{N-1} \sum_{m=0}^{N-1} \mathbf{E}\left\{\nu[n] \nu^{*}[m]\right\} e^{-j 2 \pi \alpha(n-m)}
$$

Substituting Eq. (5) into Eq. (6), it follows that

$$
\lim _{N \rightarrow \infty} N \operatorname{var}\left\{\hat{m}_{r}^{(N)}(\alpha)\right\}=\sigma^{2}\left|G\left(e^{j 2 \pi \alpha}\right)\right|^{2},
$$

where $G\left(e^{j 2 \pi \alpha}\right)=\sum_{n=-\infty}^{\infty} g[n] e^{-j 2 \pi \alpha n}$ stands for the discrete-time Fourier transform of $g[n]$.

Thus, for large enough sample size $N$, the first-order CM at $\mathrm{CF} \alpha$ with noise only presented at the receiver can be modeled as a complex Gaussian random variable with the distribution $\mathcal{C} \mathcal{N}\left(0, \sigma^{2}\left|G\left(e^{j 2 \pi \alpha}\right)\right|^{2} / N\right)$.

\section{DETECTION ALGORITHM AND PERFORMANCE ANALYSIS}

This section proposes first an algorithm for detection of AM signals by exploiting the first-order cyclostationary statistics. Then a theoretical performance analysis is carried out. The detection of AM signal is formulated as a hypothesis testing problem. The received signal is AM if a single first-order $\mathrm{CF}$ is detected. Otherwise, there is no signal (only noise) is presented at the receiver. The detection algorithm consists of the following two steps.

Step I: Based on Eq. (1), the first-order CM of the received signal is estimated based on a $N$-sample observation interval, at candidate CFs $\alpha^{\prime}$ over the range corresponding to the signal bandwidth normalized to the sampling rate. The first-order CM for AM signals are non-zero only at CF $\alpha=$ $\Delta f T_{s}$, whereas for noise the CM is statistically insignificant and close to zero.

Step II: The magnitude of the first-order CM is compared against a pre-chosen threshold $T$. If $\left|\hat{m}_{r}^{(N)}\left(\Delta f T_{s}\right)\right|>T$, there is AM signal present, otherwise not. The threshold $T$ is determined according to a given false alarm rate.

For large enough $N$, when the AM signal is present, the magnitude of CM at CF $\alpha=\Delta f T_{s}$ follows the Rician distribution with probability density function (pdf) $f_{1}(x)$

$\left|\hat{m}_{r}^{(N)}(\alpha)\right| \sim f_{1}(x)=\left\{\begin{array}{ll}\frac{x}{\sigma_{1}^{2}} e^{-\frac{x^{2}+A^{2}}{2 \sigma_{1}^{2}}} I_{0}\left(\frac{A x}{\sigma_{1}^{2}}\right) & x \geq 0 \\ 0 & x<0\end{array}\right.$,

where $I_{0}(x)$ stands for the zeroth order modified Bessel function of the first kind, and $\sigma_{1}^{2}=A^{2} \eta^{2} S_{u u}(0) / 2 N$. When there is noise only, the magnitude of CM at CF $\alpha=\Delta f T_{s}$ follows the Rayleigh distribution

$$
\left|\hat{m}_{r}^{(N)}(\alpha)\right| \sim f_{2}(x)= \begin{cases}\frac{x}{\sigma_{2}^{2}} e^{-\frac{x^{2}}{2 \sigma_{2}^{2}}} & x \geq 0 \\ 0 & x<0\end{cases}
$$

where $\sigma_{2}^{2}=\sigma^{2}\left|G\left(e^{j 2 \pi \Delta f T_{s}}\right)\right|^{2} / 2 N$.

We adopt a detector with threshold $T\left(P_{F}\right)$ such that a constant false alarm rate $P_{F}$ is achieved. The false alarm rate is given by

$$
P_{F}=\int_{T}^{\infty} f_{2}(x) d x
$$

whereas the detection probability is

$$
P_{D}=\int_{T}^{\infty} f_{1}(x) d x
$$

The performance of the detector can be evaluated only numerically. Note that the right-tail probability of the Rician random variable can be related to that of the noncentral chisquare random variable as depicted before [8] by the following equations.

$$
\operatorname{Pr}\{x>\gamma\}=Q_{\chi_{2}^{\prime 2}(\lambda)}\left(\frac{\gamma^{2}}{\sigma_{1}^{2}}\right),
$$

where $\lambda=A^{2} / \sigma_{1}^{2}$, and for $x>0$

$$
\begin{aligned}
Q_{\chi_{\nu}^{\prime}(\lambda)}(x) & =\int_{x}^{\infty} \frac{x^{\frac{\nu}{2}-1} \exp \left[-\frac{1}{2}(x+\lambda)\right]}{2^{\frac{\nu}{2}}} \sum_{k=0}^{\infty} \frac{\left(\frac{\lambda x}{4}\right)^{k}}{k ! \Gamma\left(\frac{\nu}{2}+k\right)}, \\
\Gamma(u) & =\int_{0}^{\infty} t^{u-1} e^{-t} d t .
\end{aligned}
$$

In addition to the detection of the AM signal, carrier synchronization errors can be estimated and compensated. In particular, the carrier phase offset can be estimated from the first-order CM as follows

$$
\hat{\theta}=\arg \hat{m}_{r}^{(N)}(\alpha) .
$$

The carrier frequency offset can also be recovered as $\Delta f=$ $\hat{\alpha} / T_{s}$, where $\hat{\alpha}$ is the $\mathrm{CF}$ where the first-order CM achieves the maximum.

Fig. 1 shows the magnitude of the first-order CM when $N=40$ and the SNR $=-6 \mathrm{~dB}$. The bandwidth of the modulating signal $u(t)$ is set to $8 \mathrm{KHz}$, the sampling rate is $16 \mathrm{KHz}$, the carrier frequency offset is $240 \mathrm{~Hz}$ and the carrier phase offset is $\pi / 4$. As expected, when the AM signal is present in the received signal, the first-order $\mathrm{CM}$ shows a peak in the $\mathrm{CF}$ domain at the location corresponding to the carrier frequency offset and separates itself from the noise-only set-up.

Figs. 2 - 3 illustrate the Receiver Operating Characteristics (ROC) of the proposed detection algorithm with constant false alarm rates of 0.05 and 0.01 , and when the sample size 


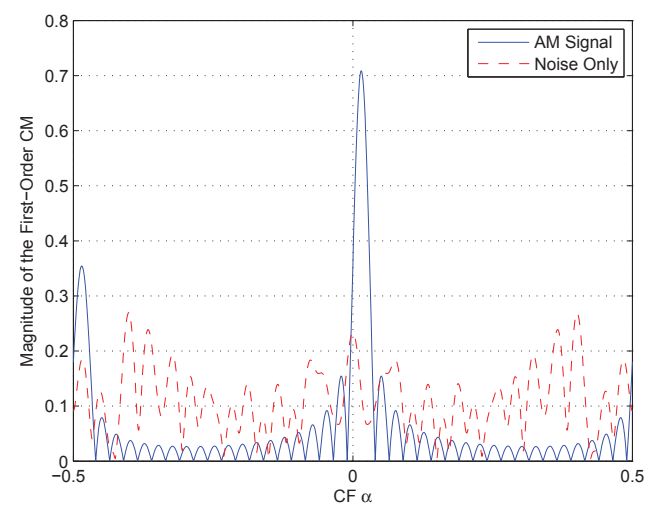

Fig. 1. The first-order $\mathrm{CM}$ with $\mathrm{CF} \alpha, \mathrm{N}=40, \mathrm{SNR}=-6 \mathrm{~dB}$.

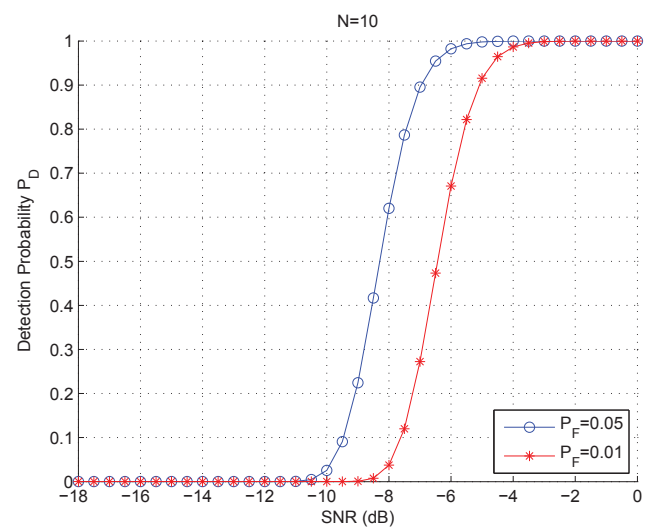

Fig. 2. Detection probabilities with $\mathrm{SNR}, \mathrm{N}=10$.

$N$ is equal to 10 and 40 samples, respectively. Ideal lowpass receive filtering and Nyquist sampling are applied and the SNR knowledge is assumed. The modulation index $\eta$ is set to 0.5 .

From Figures 2 - 3, one can easily find that the detection algorithm works well in the low SNR region that is typical for cognitive radio applications. For longer observation intervals, the power of the detector is getting better in terms of the working SNR as one would expect. Furthermore, as the sample size $N$ increases, the transitions of the detection probability curves get steeper. This can be explained by the decrease of the first-order CM estimator's variance and thus the larger separation between AM signals and noise in the CF domain. Therefore, one expects that, for large enough sample size, the detection probability curve will move to the left further and asymptotically approach the unit step.

\section{CONCLUSION}

A detection algorithm based on the first-order cyclostationarity is proposed for AM signal with relaxed a priori infor-

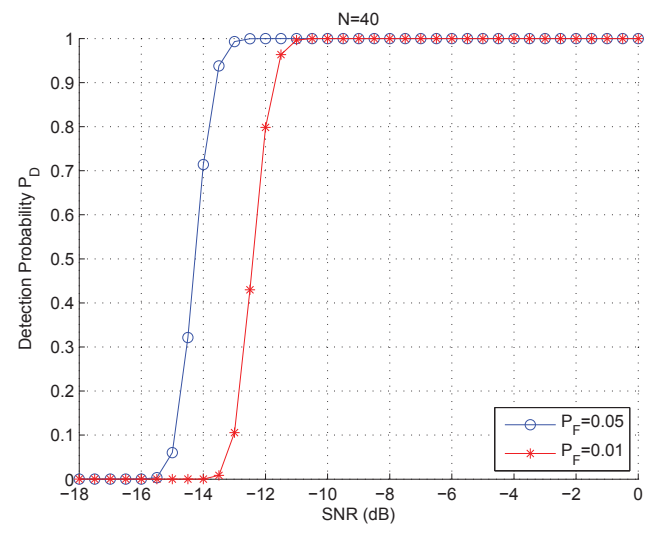

Fig. 3. Detection probabilities with SNR, N=40.

mation on signal parameters. Theoretical asymptotic analysis and numerical simulations are carried out to demonstrate the performance of the proposed algorithm. It has been shown that the algorithm performs reasonably well in the low SNR range typical for cognitive radio. Future work will be conducted for signals with different modulation formats and more complex and challenging propagation environments.

\section{REFERENCES}

[1] S. Haykin, "Cognitive radio: brain-empowered wireless communications," IEEE Journal on Selected Areas in Communications, vol. 23, no. 2, pp. 201-220, Feb. 2005.

[2] S. Haykin, D.J. Thomson, and J.H. Reed, "Spectrum sensing for cognitive radio," Proceedings of the IEEE, vol. 97, no. 5, pp. 849-877, May 2009.

[3] T. Yucek and H. Arslan, "A survey of spectrum sensing algorithms for cognitive radio applications," IEEE Communications Surveys \& Tutorials, vol. 11, no. 1, pp. 116-130, Quarter 2009.

[4] W. A. Gardner, Cyclostationarity in Communications and Signal Processing, IEEE Press, 1994.

[5] A.V. Dandawate and G.B. Giannakis, "Asymptotic theory of mixed time averages and kth-order cyclic-moment and cumulant statistics," IEEE Transactions on Information Theory, vol. 41, no. 1, pp. 216-232, Jan 1995.

[6] D. R. Brillinger and M. Rosenblatt, Asymptotic Theory of Estimates of k-th order spectra, Wiley, 1967.

[7] B. Porat, Digital Processing of Random Signals: Theory and Methods, Dover Publications, 2008.

[8] S. M. Kay, Fundamentals of Statistical Signal Processing, Volume II: Detection Theory, Prentice Hall, 1998. 